\title{
Is heart rate variability related to baroreceptors or FBN1 mutations?
}

I have read the article "A marked decrease in heart rate variability in Marfan syndrome patients with confirmed FBN1 mutations" by Cherkas et al. with interest [1]. The authors concluded that a marked decrease of heart rate variability (HRV) may be an important clinical feature in Marfan syndrome (MS) patients with confirmed FBN1 gene mutations.

We know that aortic root disease, leading to aneurysmal dilatation, aortic regurgitation, and dissection, is the main cause of morbidity and mortality in the MS [2]. Dilatation may also involve other segments of the thoracic aorta, the abdominal aorta, the root of the pulmonary artery or even the carotid and intracranial arteries. On the other hand, thoracic aortic aneurysms most often result from cystic medial degeneration that leads to weakening of the aortic wall, and MS is only one of the causes of multifactorial etiologies. The other etiological factors are chromosomal and inherited syndromes, connective tissue disorders, and inflammatory/infectious disorders [2]. In additon, previous studies have shown that pressure misregistration among baroreceptor sensor nodes, that is related to HRV [3], at different vascular bifurcations can precipitate feed-forward dysfunctions that promote thrombosis, inflammation, and vasomotor dysregulat- ion resulting in thoracic aneurysm formation $[4,5]$. Therefore, we understand that baroreceptor sensor dysfunction is not the only cause of decreased HRV, but also cause of thoracic aortic aneurysm.

Therefore, I am convinced that if the control group consisted of 30 thoracic aortic aneurysm patients without FBN1 mutations, a marked decrease of HRV might be an important clinical feature in MS patients with confirmed FBN1 gene mutations.

Conflict of interest: None declared

\section{References}

1. Cherkas A, Zhuraev R. A marked decrease in heart rate variability in Marfan syndrome patients with confirmed FBN1 mutations. Cardiol J, 2016; 23: 23-33. doi: 10.5603/CJ.a2015.0076.

2. 2014 ESC Guidelines on the diagnosis and treatment of aortic diseases. Eur Heart J, 2014; 35: 2873-2926.

3. Papaioannou VE. Heart rate variability, baroreflex function and heart rate turbulence: Possible origin andimplications. Hellenic J Cardiol, 2007; 48: 278-89.

4. Conley BR, Doux JD, Lee PY, Bazar KA, Daniel SM, Yun AJ. Integrating the theories of Darwin and Bernoulli: Maladaptive baroreceptor network dysfunction may explain the pathogenesis of aortic aneurysms. Med Hypotheses, 2005; 65: 266-272.

5. Kirsch EW, Radu NC, Allaire E, Loisance DY. Pathobiology of idiopathic ascending aortic aneurysms. Asian Cardiovasc Thorac Ann, 2006; 14: 254-260.

Sinan $\dot{I}_{\text {şcen, } M D}$

Diyarbakir Military Hospital, Yenişehir, Diyarbakir, tel: 09004122288220, fax: 09004122236237, e-mail:dr.iscen@hotmail.com 